\title{
DIABETIC RETINOPATHY- AN OVERVIEW
}

\section{Sridevi ${ }^{1}$}

${ }^{1}$ Associate Professor, Department of Ophthalmology, Rajah Muthiah Medical College and Hospital, Annamalainagar, Chidambaram.

\section{ABSTRACT}

\section{BACKGROUND}

Diabetes mellitus is a major cause of avoidable blindness in both the developing and the developed countries. Significant technological advances have taken place to improve the diagnostic accuracy of diabetic retinopathy. In the last three decades, the treatment strategies have been revised to include besides laser photocoagulation, early surgical interventions and pharmacotherapies.

\section{MATERIALS AND METHODS}

This hospital-based cross-sectional study is done on 206 Diabetic patients for the prevalence of diabetic retinopathy in the Outpatient Department of Ophthalmology, Rajah Muthiah Medical College and Hospital. Patients of all age groups with Type- I, Type- II Diabetes and Gestational Diabetes attending the OPD were studied.

\section{RESULTS}

Diabetic retinopathy is common in 51 - 60 age group. Males were commonly affected. Duration of diabetes is associated with incidence and progression of retinopathy. Majority of patients with proliferative retinopathy were on insulin.

\section{CONCLUSION}

It is a preventable cause of blindness in developing countries. Timely intervention with laser saves the patient from severe visual loss.

\section{KEYWORDS}

Diabetic Retinopathy, Diabetic Macular Oedema, Laser Photocoagulation, Pars Plana Vitreous Surgery, Intravitreal Anti-VEGF Injections.

HOW TO CITE THIS ARTICLE: Sridevi V. Diabetic retinopathy- an overview. J. Evolution Med. Dent. Sci. 2017;6(64):4660-4665, DOI: $10.14260 /$ Jemds/2017/1008

\section{BACKGROUND}

Diabetes mellitus (DM) is a major cause of avoidable blindness among the working age groups. Patients with diabetic retinopathy (DR) are 25 times more likely to become blind than non-diabetics.[1] Good glycaemic control arrests the development and progression of DR and decreases the visual loss. Technological advances have improved the diagnostic accuracy of screening methods and access of the diabetic patients to the specialist's care.

\section{Epidemiology}

India will become one of the major hubs of diabetic population during the next 2 decades according to the World Health Organisation. The prevalence of diabetes for all age groups worldwide was estimated to be $2.8 \%$ in 2000 and $4.4 \%$ in 2030 . The total number of people with diabetes is projected to rise from 171 million in 2000 to 366 million in 2030.[2] A global estimate of the prevalence of DR and the severe stages of $\mathrm{DR}^{[3]}$ from population-based studies worldwide estimated that among individuals with diabetes,

Financial or Other, Competing Interest: None.

Submission 14-07-2017, Peer Review 31-07-2017,

Acceptance 04-08-2017, Published 10-08-2017.

Corresponding Author:

V. Sridevi,

Associate Professor,

Department of Ophthalmology,

Rajah Muthiah Medical College \& Hospital,

Annamalainagar, Chidambaram-608002.

E-mail: srithithi@gmail.com

DOI: $10.14260 /$ jemds $/ 2017 / 1008$

\section{(c) $(1)(5)$}

the overall prevalence of any DR was 34.6\%, proliferative diabetic retinopathy was $7.0 \%$, diabetic macular oedema was $6.8 \%$ and vision threatening diabetic retinopathy was $10.2 \%$.

In two studies from South India, the prevalence rates of DR in type 2 diabetic patients were $34.1 \%$ and $37 \% .{ }^{[4]}$ In the Chennai Urban Rural Epidemiology Study, they evaluated urban sample of diabetic patients and estimated the overall prevalence of DR as $17.6 \% .{ }^{[5]}$

\section{Pathophysiology}

There is a complicate interplay of various factors in the pathogenesis of diabetic retinopathy such as bio-chemical mechanisms, rheological changes and structural changes. Bio-chemical mechanism includes prolonged hyperglycaemia, excess sorbitol formation,[6] protein kinase C activation ${ }^{[7]}$ and vascular endothelial growth factor release. Prolonged hyperglycaemia ${ }^{[8]}$ is the major aetiologic agent in the microvascular complications of diabetes mellitus. Chronic hyperglycaemia causes accelerated oxidative stress in cells resulting in toxic end products. [9] Rheological changes includes increased platelet adhesion and aggregation, and increased rouleaux formation and reduced deformability of red blood cells. Structural changes include capillary basement membrane thickening, loss of microvascular intramural pericytes and breakdown of blood retinal barrier. However, the final metabolic pathway causing DR is still unknown. 


\section{Risk Factors of Diabetic Retinopathy Level of Glycaemia}

Hyperglycaemia is a strong factor in the development and progression of diabetic retinopathy. Benefits of better control continue to manifest even after non-proliferative and proliferative diabetic retinopathy has developed. Elevated glycosylated haemoglobin (HbA1c) is a strong factor for the progression of high-risk proliferative diabetic retinopathy.[10]

\section{Serum Lipids}

Elevated levels of serum cholesterol is associated with increased severity of hard exudates.[11] Elevated serum triglyceride levels are associated with an increased risk of developing high risk and decreased visual acuity.

\section{Blood Pressure}

Intensive control of blood pressure slows down the progression of retinopathy and reduces the risk of other microvascular and macrovascular complications of diabetes mellitus ${ }^{[2]}$ Abnormal systolic and diastolic blood pressures are associated with the severity of retinopathy in both type I and type II disease.

\section{Duration of Diabetes}

Duration of diabetes is a significant risk factor for the development of diabetic retinopathy. After 20 years of diabetes, all the type I and $>60 \%$ of type II patients have some degree of retinopathy.

\section{Pregnancy}

Retinopathy is accelerated during pregnancy, because of pregnancy itself or the changes in the metabolic control. [13]

\section{Genetic Factors}

Relationship between HLA antigens expressed on the cell surface and the presence of retinopathy has already been documented. HLA- DR phenotypes 4/0, 3/0 and XX expression is associated with increased proliferative retinopathy. ${ }^{[14]}$

\section{Ocular Factors}

Myopia reduces the prevalence and severity of diabetic retinopathy. Retinochoroidal scarring from trauma or inflammatory disease reduces the prevalence of retinopathy by decreasing the retinal metabolism and thereby decreasing the need for oxygen and the release of vasoproliferative factors.

Clinical Features of Diabetic Retinopathy, NonProliferative and Proliferative Diabetic Retinopathy

Non-proliferative diabetic retinopathy (NPDR) is characterised by the presence of: (i) Microaneurysms, which are the first clinically detectable lesions of DR located in the inner nuclear layer of the retina, (ii) Dot and blot haemorrhages, which are located in the middle retinal layers, (iii) Hard exudates, which are located between the inner plexiform and inner nuclear layer of the retina, (iv) Vascular changes such as beading, looping and sausage-like segmentation of the veins, (v) Cotton wool spots, also called soft exudates or nerve fibre infarcts result from capillary occlusion of the retinal nerve fibre layer, (vi) Intraretinal microvascular abnormalities (IRMA) which are dilated capillaries that seem to function as collateral channels are frequently seen adjacent to the areas of capillary closure, (vii) Retinal oedema characterised by accumulation of fluid between the outer plexiform layer and inner nuclear layer, which may later involve the entire layers of the retina.

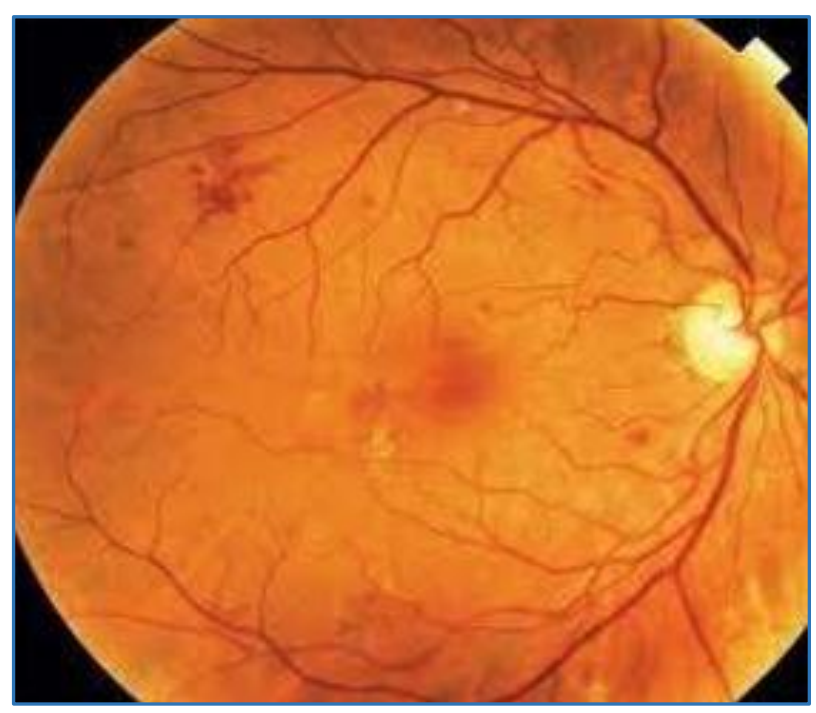

Figure 1. NPDR with CSME

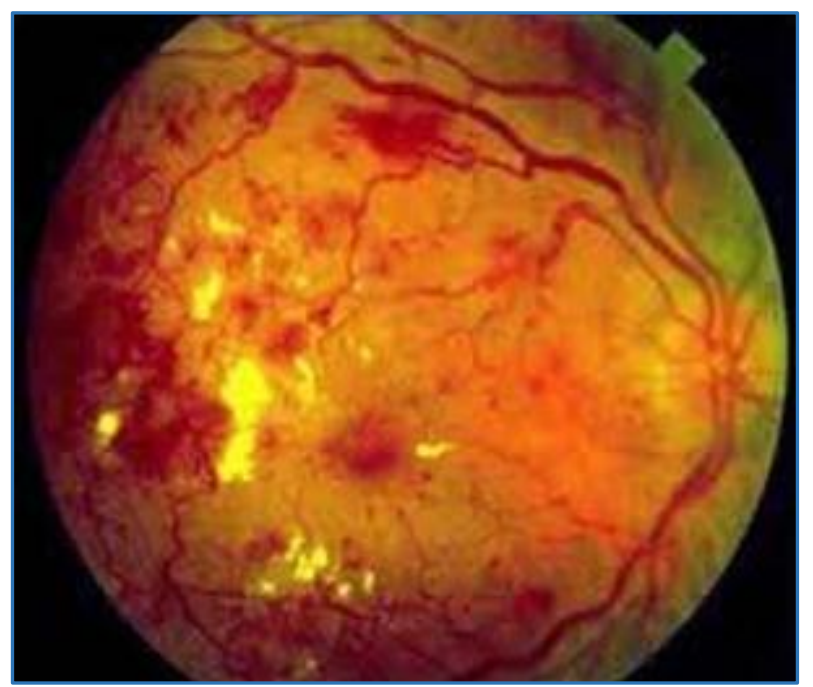

Figure 2. PDR

In the natural course, approximately $50 \%$ of patients with very severe NPDR progress to proliferative diabetic retinopathy (PDR) within 1 year.[15] PDR is characterised by the presence of neovascularisation. New vessels may proliferate on the optic nerve head (new vessels at disc- NVD) and along the course of the major vascular arcades (new vessels elsewhere- NVE). The new vessels mostly grow along the posterior hyaloid and sudden vitreous contraction may result in rupture of these fragile vessels. When the vitreous detachment occurs, the new vessels are pulled anteriorly along with the underlying retina resulting in tractional retinal detachment. On the other hand vitreous might detach completely without any pull on the retina and new vessels regress, thus resulting in the development of an end-stage disease.

The Early Treatment Diabetic Retinopathy Study (ETDRS) ${ }^{[16]}$ has classified NPDR into mild, moderate, severe 
and very severe and PDR into early PDR and high-risk PDR. This is as follows-

A. Moderate NPDR: Haemorrhages and/or microaneurysms, presence of soft exudates, venous beading, IRMA definitely present, definition not met for C, D, E or F.

B. Mild NPDR: Presence of at least one microaneurysm, definition not met for B, C, D, E or F.

C. Severe NPDR: Haemorrhages and/or microaneurysms in all four quadrants or venous beading in two or more quadrants or IRMA in at least one quadrant, definition not met for D, E or F.

D. Very severe NPDR: Any two or more of the changes seen in severe NPDR, definition not met for $\mathrm{E}$ or F.

E. Early PDR: Presence of new vessels, definition not met for F.

F. High-risk PDR: Includes any of the following characteristics- neovascularisation of disc (NVD) $>1 / 3^{\text {rd }}$ to $1 / 4^{\text {th }}$ disc diameter, NVD $<1 / 3^{\text {rd }}$ to $1 / 4^{\text {th }}$ disc diameter with vitreous/ pre-retinal haemorrhage, NVE with vitreous/ pre-retinal haemorrhage. High-risk characteristics (HRC) were defined by Diabetic Retinopathy Study, as the patient if not treated urgently is at a high risk of severe visual loss.

\section{Diabetic Macular Oedema}

Macular oedema or retinal thickening is an important manifestation of DR and the most common cause of moderate visual loss. Diabetic macular oedema patients were categorised into clinically significant macular oedema (CSME) ${ }^{[17]}$ or non-CSME by ETDRS. CSME includes any one of the following lesions-

1. Retinal thickening at or within 500 microns from the centre of macula.

2. Hard exudates at or within 500 microns from the centre of macula associated with thickening of the adjacent retina.

3. An area or areas of retinal thickening at least one disc area in size, at least a part of which is within one disc diameter of the centre of macula.

The intraretinal fluid comes from leaking microaneurysms or diffuses from capillary incompetence areas. Diabetic macular oedema is retinal thickening within two disc diameters of the centre of macula.

\section{MATERIALS AND METHODS}

This hospital-based cross-sectional study is done on 206 Diabetic patients for the prevalence of diabetic retinopathy in the Outpatient Department of Ophthalmology, Rajah Muthiah Medical College and Hospital. Patients of all age groups with Type- I, Type- II Diabetes and Gestational Diabetes attending the OPD were studied during a one-year period from October 2015 to September 2016. Patients will be selected on the basis of criteria as follows.

\section{Inclusion Criteria}

1. All cases of Diabetics of all age groups who came to OPD, Rajah Muthiah Medical College and Hospital were included.

2. Type- I, Type- II Diabetes and Gestational Diabetes were also included.

\section{Exclusion Criteria}

1. Non-compliant, Immunocompromised patients.

2. Patients with hypertension, cardiac disease, renal disease.

\section{Materials Used}

1. Snellen's visual acuity chart.

2. Schiotz tonometer.

3. Carl-Zeiss slit lamp.

4. Direct ophthalmoscope.

5. Indirect ophthalmoscope.

6. + 78D and 90D lens.

A comprehensive eye exam is done along with visual acuity and detailed anterior segment and fundus examination. Diabetic retinopathy was classified as no retinopathy, mild non-proliferative retinopathy (NPDR), moderate NPDR, severe NPDR, very severe NPDR, early PDR, high-risk PDR and presence or absence of CSME.

\section{Statistical Methods}

The parameters in this case series were compared by applying independent ' $t$ ' test, i.e. test based on comparison between two sample means, i.e. non-proliferative retinopathy and proliferative retinopathy. The results were obtained using SPPS 11 version package. The data collected were analysed on the basis of descriptive statistics, mean and standard deviation. The graphs and diagrams were also used to represent the data. Microsoft word and excel had been used to generate graphs and tables.

\section{Management \\ Investigations}

Diabetic retinopathy is essentially a clinical diagnosis. Slit lamp biomicroscopy, dilated fundus evaluation with a direct ophthalmoscope and indirect ophthalmoscope or contact/ non-contact slit lamp biomicroscopic examination are essential in the diagnosis of DR. However, several ancillary investigations are required to aid the diagnosis, plan and execute the treatment and to document the lesions for research purposes. Stereoscopic fundus photographs may be required for research purposes and are especially useful for the assessment of macular oedema.

\section{Laser Photocoagulation}

The treatment depends on the Type and Severity of Retinopathy, Non-Proliferative Retinopathy

For mild and moderate NPDR, strict adherence to normal levels of glycaemia, blood pressure and lipid status is the mainstay of effective treatment. Scatter laser photocoagulation is generally not recommended. The Early Treatment Diabetic Retinopathy Study[18] and the Diabetic Retinopathy Study recommend photocoagulation as the treatment of choice for severe and progressive form of retinopathy and clinically significant macular oedema.

\section{Severe Non-Proliferative Diabetic Retinopathy}

For severe NPDR, scatter laser treatment is appropriate when the disease process is progressing rapidly and when close follow-up of patients are unlikely.

\section{Macular Oedema}

ETDRS demonstrated that retinal laser therapy applied to macula reduces the risk of substantial worsening of vision by 
$50 \%$. Parameters for focal treatment should be the spot size about $50-100 \mu \mathrm{m}$ of $\leq 0.1 \mathrm{~s}$ duration and power sufficient to cause blanching of microaneurysm and retinal pigment epithelium.

\section{Pars Plana Vitreous Surgery}

Diabetic retinopathy vitrectomy study[19] randomised 370 eyes with extensive neovascularisation and visual acuity of $20 / 400$ or better into two groups of early vitrectomy or observation alone. The results indicate that such patients probably do not benefit from early vitrectomy. They should be observed closely, so that vitrectomy when needed can be undertaken promptly. This group also studied diabetic eyes with vitreous haemorrhage and visual acuity less than $5 / 200$ for 6 months and randomised these into two groups of those who received immediate surgery and those whose surgery was deferred for another 6 months. The study recommended early surgery in type 1 diabetic patients, more so in bilateral cases and one-eyed patients.

\section{Other Strategies in Diabetic Retinopathy Management}

Diabetes Control and Clinical Trial (DCCT) ${ }^{[20]}$ showed that in intensively treated group the risk of onset of retinopathy was reduced by $76 \%$, risk of progression of retinopathy by $63 \%$, risk of development of CSME by $23 \%$ and the need for laser treatment by $56 \%$ compared to the conventional group. This benefit persisted even 4 years after initiation of intensive therapy.

United Kingdom Prospective Diabetes Study (UKPDS) showed that in intensive blood pressure control group, there was a $34 \%$ and $47 \%$ reduction in risk of DR progression and moderate visual acuity loss respectively compared to the control group after a median followup of 8.4 years.

ETDRS identified elevated levels of serum cholesterol and low-density lipoproteins as independent risk factors for the development of hard exudates, which is a major risk factor leading to subfoveal fibrosis.

In patients with refractory CSME, intravitreal administration of corticosteroids showed to be useful. Many trials have been done reporting the efficiency of intravitreal anti-VEGF agents.[21] Currently, several drug delivery modalities are in clinical trials to investigate their efficacy.

\section{Screening for Diabetic Retinopathy}

Ophthalmoscopy is the most commonly used technique to screen for DR. When performed by an ophthalmologist, specificity of direct and indirect ophthalmoscopy was high, but the sensitivity was low.

The digital imaging makes fundus photography easier and more widely accessible. Single-field fundus photography with interpretation by trained readers could serve as a screening tool to identify patients with DR.

The automated retinal image screening ${ }^{[22]}$ for diabetic retinopathy had a high sensitivity in high and medium quality images and this software hold promise in future screening programs.

\section{RESULTS}

In our study 206 diabetic patients reported to eye OPD, out of which 75 visually symptomatic patients were taken into study with varying severity of retinopathy. When evaluated the following levels when exceeded were considered as abnormal.

- $\quad$ Fasting blood glucose $=110 \mathrm{mg} / \mathrm{dL}$

- $\mathrm{HbA1c}=6 \%$

- Blood urea $=40 \mathrm{mg} / \mathrm{dL}$

- $\quad$ Serum creatinine $>1.5 \mathrm{mg} / \mathrm{dL}$

- $\quad$ Blood pressure $=140 / 90 \mathrm{mmHg}$

\begin{tabular}{|c|c|c|}
\hline Age & & Total \\
\hline \multirow{2}{*}{ Upto 30} & Count & 3 \\
\cline { 2 - 3 } & \% of DR & $4.0 \%$ \\
\hline \multirow{2}{*}{$31-40$} & Count & 4 \\
\cline { 2 - 3 } & $\%$ of DR & $5.3 \%$ \\
\hline \multirow{2}{*}{$41-50$} & Count & 22 \\
\cline { 2 - 3 } & \% of DR & $29.3 \%$ \\
\hline \multirow{2}{*}{$51-60$} & Count & $33.3 \%$ \\
\cline { 2 - 3 } & \% of DR & 21 \\
\hline \multirow{2}{*}{$61-70$} & Count & $\mathbf{7 5}$ \\
\cline { 2 - 3 } & \% of DR & $\mathbf{1 0 0 \%}$ \\
\hline \multirow{2}{*}{ Total } & Count \\
\cline { 2 - 3 } & \% of DR \\
\hline \multicolumn{2}{|c|}{ Table 1. Age Distribution } \\
\hline
\end{tabular}

In the study, predominate age group affected is $51-60$ years followed by 41 - 50 years. The least involved was less than 40 years.

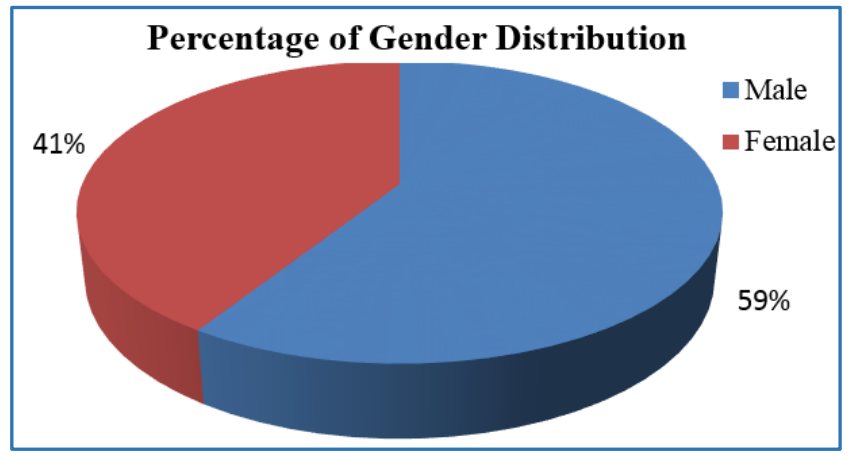

Figure 3. Percentage of Gender Distribution

In this study, males were commonly affected than females. The sex ratio between males and females was 1.5:1.

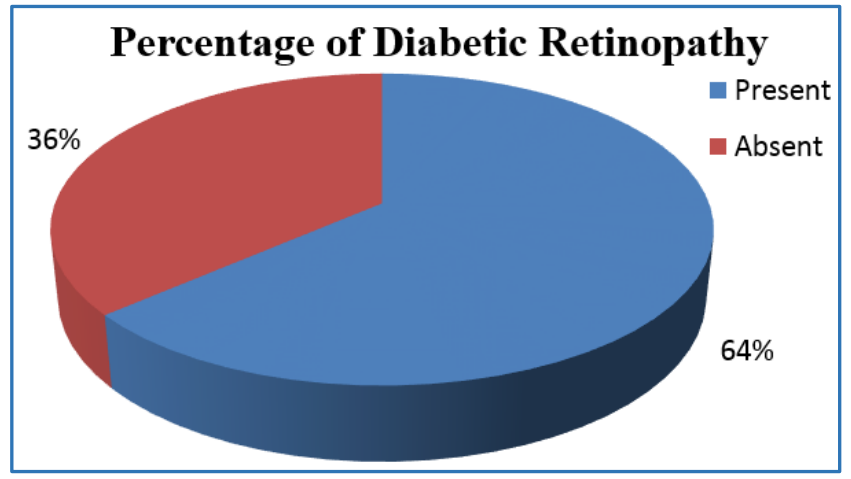

Figure 4. Percentage of Diabetic Retinopathy

Two-thirds of the patients with diabetes did not show any evidence of retinopathy at all. 


\begin{tabular}{|c|c|c|}
\hline DR & Number & $\mathbf{\%}$ \\
\hline No Retinopathy & 131 & $64 \%$ \\
\hline NPDR & 44 & $21 \%$ \\
\hline NPDR with CSME & 12 & $6 \%$ \\
\hline CSME & 14 & $7 \%$ \\
\hline PDR & 5 & $2 \%$ \\
\hline Table 2. Distribution of Diabetic Retinopathy \\
\hline
\end{tabular}

$64 \%$ of patients had no retinopathy and $2 \%$ had proliferative retinopathy in our study.

\begin{tabular}{|c|c|c|c|}
\hline Type of DR & & FBS & HbA1c \\
\hline \multirow{2}{*}{ NPDR } & Mean & 193.37 & 9.132 \\
\cline { 2 - 4 } & SD & 69.94704 & 2.9035 \\
\hline \multirow{2}{*}{ PDR } & Mean & 278.4 & 12.32 \\
\cline { 2 - 4 } & SD & 44.39369 & 2.33 \\
\hline 't' & 3.96 & 2.91 \\
\hline 'P' Value & 0.00005 & 0.00196 \\
\hline \multicolumn{3}{|c|}{ Table 3. Fasting Blood Glucose, HbA1c and Diabetic } \\
Retinopathy \\
\hline
\end{tabular}

By applying ' $t$ ' test, $p$ value $(<0.05)$ was significant for FBS and HbA1c.

\section{DISCUSSION}

The diabetics attending eye OPD were enrolled and studied for the pattern of presentation of diabetic retinopathy. In this study, the predominant age group affected is 51 - 60 years (33\%). This was conformed in a study done by Dandona et $\mathrm{al}^{23}$ Agarwal et $\mathrm{al}^{24}$ and Klein et al. Most of the male sex was associated with severe retinopathy, which correlated with a study by Bodansky et $\mathrm{al}^{25}$ and Rema et al. Among 75 patients, $13 \%$ had maculopathy similar to study done by Khandekar et al 2003.26 The other types of retinopathy and its incidence correlated with the study conducted by Agarwal et $\mathrm{al}^{24}$ and Narendran et al. ${ }^{27}$ In individuals with long standing diabetics, there is increased risk for DR occurrence which correlated with a study by Zander et al. ${ }^{28}$ Hyperglycaemia was an associated factor in all the groups as shown by Klein et al. Treatment with insulin is associated with more severe progressive retinopathy, Agarwal et al.

\section{CONCLUSION}

In this case series, Diabetic retinopathy was common after 50 years of age and was common in males. NPDR was the most common form. Increased duration of diabetes was associated with increased risk of retinopathy along with increased FBS and increased HbA1c. High blood pressure was strongly associated with retinopathy.

Of the patients reported, $36 \%$ had diabetic retinopathy with NPDR as common form. Maculopathy occurred in 13\% of the diabetics. Most of the patients with PDR had Type 1 diabetes.

There was increased risk of NPDR and CSME in Type 2 diabetes. Majority of NPDR had presenting visual acuity greater than $6 / 36$.

Majority of patients with PDR were on insulin. The present review outlines the magnitude of the problem in India, conventional and current strategies to manage the potentially blinding complications of DM.

\section{REFERENCES}

[1] National society to prevent blindness. New York: National society to prevent blindness 1980:1-46.
[2] Wild S, Roglic G, Green A. Global prevalence of diabetes: estimates for the year 2000 and projections for 2030. Diabetes Care 2004;27(5):1047-53.

[3] Yaw JW, Rogers SL, Kawasaki R, et al. Global prevalence and major risk factors of diabetic retinopathy. Diabetic care 2012;35(3):556-64.

[4] Rema M, Ponnaiya M, Mohan V. Prevalence of retinopathy in non-insulin dependent diabetes mellitus at a diabetes centre in southern India. Diabetes Res Clin Pract 1996;34(1):29-36.

[5] Rema M, Premkumar S, Anitha B, et al. Prevalence of diabetic retinopathy in urban India: the Chennai Urban rural epidemiology study (CURES) eye study. Invest Ophthalmol Vis Sci 2005;46(7):2328-33.

[6] Enden MKV, Nyengaard JR, Ostrow E, et al. Elevated glucose levels increase retinal glycolysis and sorbital pathway metabolism. Implications for diabetic retinopathy. Invest Ophthalmol Vis Sci 1995;36(8):1675-85.

[7] Xia P, Inoguchi T, Kern TS, et al. Characterisation of the mechanism for the chronic activation of diacylglycerol-protein kinase $\mathrm{C}$ pathway in diabetes and hypergalactosemia. Diabetes 1994;43(9):1122-9.

[8] Maitra A. In: The endocrine system: Robbins and Cotran pathologic basis of diseases. $8^{\text {th }}$ edn. Published by Elsevier 2010:1131-46.

[9] Murata T, Nakagawa K, Khalil A, et al. The relation between expression of vascular endothelial growth factor and breakdown of the blood retinal barrier in diabetic rat retinas. A Journal of Technical Methods and Patholog 1996;74(4):819-25.

[10] Klein R, Klein BE, Moss SE, et al. The Wisconsin epidemiologic study on diabetic retinopathy II. Prevalence and risk of diabetic retinopathy when age at diagnosis is less than 30 years. Arch Ophthalmol 1984;102(4):520-6.

[11] Chew EY, Klein ML, Remaley NA, et al. Association of elevated serum lipid levels with retinal hard exudate in diabetic retinopathy. Early treatment diabetic retinopathy study (ETDRS) report 22. Arch Ophthalmol 1996;114(9):1079-84.

[12] King P, Peacock I, Donnelly R. The United Kingdom prospective diabetic study (UKPDS): clinical and therapeutic implications for type 2 diabetes. $\mathrm{Br} \mathrm{J}$ Clin 1999;48(5):643-8.

[13] Chew EY, Mills JL, Metzger BE, et al. Metabolic control and progression of retinopathy. The diabetes in early pregnancy study. National institute of child health and human development diabetes in early pregnancy study. Diabetes Care 1995;18(5):631-7.

[14] Baker RS, Rand LI, Krolewski AS, et al. Influence of HLA-DR phenotype and myopia on the risk of no proliferative and proliferative diabetic retinopathy. American Journal of Ophthalmology 1986;102(6):693-700.

[15] Kohner EM, Aldington SJ, Stratton IM, et al. United Kingdom prospective diabetes study 30, diabetic retinopathy at diagnosis of non-insulin-dependent diabetes mellitus and associated risk factors. Arch Ophthalmol 1998;116(3):297-303. 
[16] Turner R, Holman R. Tight blood pressure control and risk of macrovascular and microvascular complications in type 2 diabetics: UKPDS 38. BMJ 1998;317:703-13.

[17] Photocoagulation for diabetic macular edema. Early treatment diabetic retinopathy study report number 1. Early treatment diabetic retinopathy study research group. Arch ophthalmol 1985;103(12):1796-806.

[18] Techniques for scatter and local photocoagulation treatment of diabetic retinopathy. Early treatment diabetic retinopathy study report number 3 . The early treatment diabetic retinopathy study research group. Int Ophthalmol Clin 1987;27(4):254-64.

[19] Aaberg TM. Clinical results in vitrectomy for diabetic traction retinal detachment. American Journal of Ophthalmology 1979;88(2):246-53.

[20] Nathan DM. The diabetes control and complications trial/epidemiology of diabetes interventions and complications study at 30 years: overview. Diabetes Care 2014;37(1):9-16.

[21] Domalpally A, Ip MS, Ehrlich JS. Effects of intravitreal ranibizumab on retinal hard exudate in diabetic macular edema: findings from the RIDE and RISE phase III clinical trials. Ophthalmology 2015;122(4):779-86.
[22] Roy R, Lob A, Pal BP, et al. Automated diabetic retinopathy imaging in Indian eyes: a pilot study. Indian J Ophthalmol 2014;62(12):1121-4.

[23] Dandona L, Dandona R, Naduvilath T, et al. Population based assessment of diabetic retinopathy in an urban population in southern India. $\mathrm{Br} \mathrm{J}$ Ophthalmol 1999;83(8):937-40.

[24] Agarwal RP, Ranka M, Ghugare BW, et al. Prevalence of diabetic retinopathy in type 2 diabetes in relation to risk factors. Int J Diab Dev countries 2003;23:16-9.

[25] Bodansky HJ, Cudsworth AG, Whitelocke RA, et al. Diabetic retinopathy and its relation to type of diabetes: review of a retinal clinic population. British Journal of Ophthalmology 1982;66(8):496-9.

[26] Khandekar R, Lawatii JA, Mohammed AJ, et al. Diabetic retinopathy in Oman: a hospital based study. Br J Ophthalmol 2003;87(9):1061-4.

[27] Narendran V, John RK, Raghuram A, et al. Diabetic retinopathy among self-reported diabetics in southern India: a population based assessment. $\mathrm{Br} \mathrm{J}$ Ophthalmol 2002;86(9):1014-8.

[28] Zander E, Herfurth S, Bohl B, et al. Maculopathy in patients with diabetes mellitus type 1 and type 2: associations with risk factors. $\mathrm{Br} \mathrm{J}$ Ophthalmol 2000;84(8):871-6. 